\title{
Aspectos metodológicos
}

Los contenidos del presente libro se encuentran fundamentados en los resultados de trabajos de investigación desarrollados por Maestrantes y sus correspondientes Directores, a través de los hallazgos alcanzados y evidenciados en sus trabajos de grado, en virtud del Convenio de Regalías del Departamento de Sucre para la financiación de sus proyectos. En ese sentido, debido a la diversidad de metodologías que estos presentan, se hace necesario referenciar tanto a los Autores como sus Directores de Tesis, así como las metodologías utilizadas para desarrollar estos contenidos.

El Capítulo 1 presenta componentes de Índice de Competitividad y la caracterización de las regiones económicas del Departamento de Sucre, como un apartado introductorio que proporciona una línea base desde donde se abordan diferentes aristas que se podrán evidenciar en el tratamiento de cada uno de los Capítulos posteriores. El análisis del Índice de Competitividad de Sucre se presentó a partir de los resultados del Índice Departamental de Competitividad (2019), realizado por el Consejo Privado de Competitividad y la Universidad del Rosario. En este sentido, las características de las Regiones y los datos económicos del Departamento, son presentadas de acuerdo con las fuentes oficiales y resultados investigativos previos, realizados por el Banco de la República, en los que se aborda el ámbito de la economía departamental.

Las dinámicas económicas y caracterización de los renglones económicos más representativos de la economía sucreña corresponden al Capítulo 2. Este le permite al lector tener una aproximación con los datos, para convertirse en el prólogo de capítulos posteriores, en virtud de la comprensión de los mismos y su posible conexión con las posibilidades de identificar potencial de transformación en el Departamento. Para lo cual, se presenta a cada uno de los Subsectores que se van a tratar, desde los aportes realizados por cada uno de los Autores.

Cabe destacar que, para tales fines, los aportes de Raúl Acosta, Jorge Del Río, Javier Prieto y Andrés Vergara Narváez, cuyas contribuciones sobre la Competitividad agrícola de Sucre, con especial énfasis en el conflicto 
de vocación de la tierra del Departamento, enriquecieron este proyecto en gran medida.

Tomando en cuenta que se tocan dos temáticas profundas, como son la Competitividad y la Prospectiva en términos regionales y su respectiva evolución, el Capítulo 3 expone los referentes teóricos y empíricos para contextualizar, en mejor medida, los resultados de las investigaciones. Para tales fines, se mencionaron los Autores más relevantes en cada temática, con el fin de que el lector pueda conectar los resultados obtenidos de los Sectores Económicos del Departamento con la teoría, y, de esa manera, este pueda tener una visión holística de la situación económica en el Departamento, con fundamento en lo teórico y empírico. Lo anterior, evidencia la contribución de los Directores de Trabajo de Grado Piedad Martínez y Verónica Tordecilla.

El Capítulo 4 presenta hallazgos de los retos y desafíos que el Departamento de Sucre tiene frente a los procesos de Competitividad, para lo cual, los renglones económicos más representativos del Departamento de Sucre, fundamentados en la caracterización de estos Sectores fueron realizados previamente.

Los retos y desafíos para mejorar los Niveles de Competitividad en los diferentes Subsectores; la Yuca Industrial en el Municipio de San Pedro, Sucre, desarrollado por Engler de Jesús Ariza García; en el caso de la Competitividad de la Cadena de Valor del Subsector Cárnico-bovino de la Subregión Sabanas del Departamento de Sucre, los hallazgos los presenta Carmen Danith Aguado; en lo relacionado con el Sector Lácteo y de Queso desde el Municipio de Buenavista fue abordado por Gloria Teresa Lozano Rivera; en el Sector de las Artesanías, se resalta que existe información limitada y escasa por la alta informalidad, por lo tanto, para establecer las necesidades particulares de los Artesanos en el Departamento de Sucre, en términos de Competitividad, se realizó trabajo exploratorio que permitiera conocer, en primera instancia, las debilidades y fortalezas de estos Artesanos.

En la Tejeduría en Hilos, en el Municipio de Morroa, Sucre, la información fue recabada y analizada por Julio Alberto Guzmán Rodríguez. Por su parte, los desafíos para el Sector de la Caña Flecha en Sampués fueron planteados por Erika Patricia Ruiz Carta. 
El análisis del Sector Hotelero fue elaborado gracias a los aportes de María Alejandra Santis Puche, considerando lo planteado por el Plan de Desarrollo Turístico de Sucre 2016-2020 propuesto por la Secretaría de Planeación de la Gobernación del Departamento y estudio particular del Sector.

Al final, se proponen unos retos y desafíos en términos del Sector Apícola y la Educación Emprendedora en el Departamento, partiendo de una metodología Prospectiva, la cual dictamina la construcción de escenarios futuros, trabajándolos desde el presente, expuestos en el Capítulo 5. El apartado Apícola cuenta con la participación de Isabel Inés Meza Herazo, mientras que la Educación Emprendedora de Sucre desde la Prospectiva se realizó a partir de lo propuesto por Liliana Isabel Guzmán Orozco. 